\title{
SwellFit: Developing A Wearable Sensor for Monitoring Peripheral Edema
}

\author{
Sunyoung Kim \\ Rutgers University \\ sunyoung.kim@rutgers.edu
}

\author{
Yasha Iravantchi \\ Carnegie Mellon University \\ ysi@cs.cmu.edu
}

\author{
Krzysztof Z. Gajos \\ Harvard University \\ kgajos@eecs.harvard.edu
}

\begin{abstract}
Peripheral edema is a swelling of the legs, feet, or hands due to the accumulation of excessive fluid in the tissues. For patients with some chronic diseases, peripheral edema is a crucial indicator of onset or exacerbation of the condition. Thus, early detection of peripheral edema is important for timely diagnosis of associated diseases. However, existing techniques for edema assessment are a subjective measurement for which a human operator estimates the amount of swelling using a tape measure or by pressing the swollen area with the tip of an index finger. As a systematic approach to assessing peripheral edema, we develop SwellFit, an experimental prototype of a novel wearable technology that monitors peripheral edema by tracking changes in ankle curvature. Through a series of proof-of-concept experiments, we demonstrate that SwellFit detects ankle swelling even in the presence of substantial noise in the raw sensor readings.
\end{abstract}

\section{Introduction}

Peripheral edema is swelling or puffiness of peripheral part of the body, usually happening in the feet, ankles or hands. Edema occurs when tiny blood vessels in the body leak fluid so that the fluid builds up in surrounding tissues. Many things in daily activities can cause edema, including sitting or standing still for too long, excessive salt intake, and medicines. When any of these things cause edema, the swelling is often temporary and clears up by itself. Edema, however, can also associate with more serious underlying medical conditions, such as diabetes, heart failure, and kidney disease. For patients suffering from these conditions, peripheral edema is considered as a crucial indicator of onset or exacerbation of the condition. Thus, early detection of peripheral edema is important for patients to recognize underlying conditions of some diseases in time and make proper action to prevent further clinical dysfunction or deterioration [1].

Among various medical conditions, congestive heart failure (CHF) is one of the most prevalent diseases for which edema is used as an important physical indicator of worsening of the condition. When the heart weakens and pumps blood less effectively due to CHF, fluid can slowly build up, causing leg edema. As the most common diagnosis in patients 65 and older in the United States, CHF is known as a leading cause of hospitalization and readmission [9] which imposes a huge financial burden and degraded quality of care [17]. Thus, preventable hospital readmission is an increasingly prominent target in both policy and medical discussions. While a variety of direct measures have been taken into consideration to empower patients to monitor the condition at home and to reduce or prevent readmission, edema has not been considered as a means of patient self-measurement perhaps because there is no easy and systematic way for patients to measure edema.

Currently, the most widely used technique for edema measurement is a subjective assessment for which a clinical examiner applies pressure with an index finger to a single location on the patient's ankle and examine the amount of depression. This technique captures the amount of depression and the time needed for the skin to return to its original appearance as a single edema score ranging from 0 to 4 [21]. While this classic method is commonly used, it has not been proven to be a sufficiently objective, reliable, or sensitive assessment of edema [7]. More rigorous, objective, and yet easy way to assess edema is needed when careful evaluation of edema is required as part of patient-centric care practices.

With the advent of IoT and the availability of sophisticated sensors that can monitor a myriad of biometric parameters, wearable technology has gained the interest of researchers and clinicians due to the benefits that could be associated with long-term monitoring of individuals for healthcare [6] As a result, a great number of wearable systems that are suitable for clinical applications emerged in the past few years. They range from simple monitoring of daily activities for the purpose of assessing mobility and level of independence in individuals, to capturing patients' physiological signals to facilitate clinical evaluation, and to integrating miniature sensors to support performance of motor tasks that are otherwise unable 
to accomplish [3] However, sensor-based measurement of edema to overcome the shortcomings of manual assessments has received relatively less attention to date despite its importance. Only recently, few studies have attempted to develop wearable technologies to systematically assess edema using a stretch sensor that measures the circumference of an ankle (e.g., $[11,12])$.

In this paper, we introduce SwellFit, an experimental prototype that may inform the final design of a novel wearable technology for continuous assessment of edema by monitoring the changes in the curvature of an ankle using a flex sensor. The goal of this paper is to describe and demonstrate the feasibility of SwellFit. SwellFit is equipped with a combination of wearable sensors and hardware, intelligent data processing, and a smartphone application to provide a reliable and user-friendly system. The primary contribution of this paper comes in three parts:

- A wearable technology to continuously monitor peripheral edema with unique hardware design;

- Signal processing techniques to detect a signal of edema from tracking relative changes in the ankle curvature;

- Feasibility testing of SwellFit in a comprehensive experimental procedure.

\section{Background}

\subsection{Existing edema measurement}

Two prevalent methods of edema assessment in clinical practices include pitting and tape measure. For pitting, a clinician applies pressure on the skin with the tip of an index finger and estimates the amount of edema based on the amount of time it takes for the pitting to come back to its original level after a finger is removed. The amount of depression indicates the amount of pitting (Figure 1). Another method is to measure a circumference of an ankle using a tape measure, which requires making sure to measure at the same point of an ankle each and every time. These approaches must take place clinically, and thus is burdensome and expensive. Most importantly, in some cases these methods are unreliable due to its subjectivity and fail to detect edema in time.

\subsection{Wearables for Physiological Monitoring}

For many healthcare use cases of wearable sensors, it is highly desirable to have sensors capable of directly monitoring the physiology of the wearer in real-time. These sensors can measure biological, chemical or physical phenomena to assess physiology. The technology challenge is how to maintain consistent

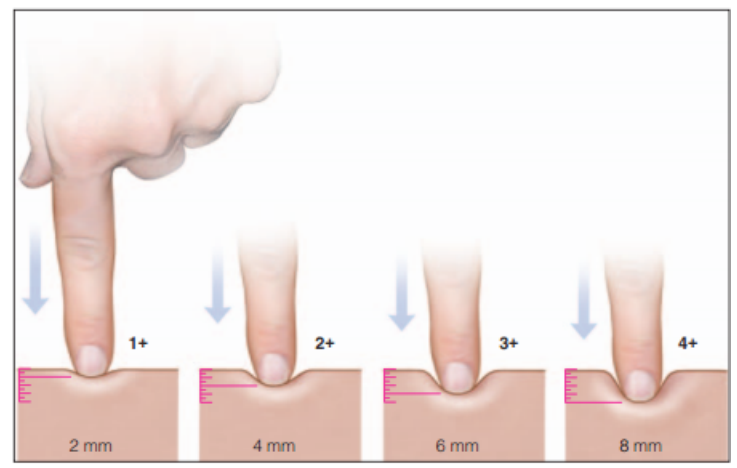

Figure 1. Pitting edema: Dent depth and duration from 1 to 4 (image from http://www.med-health.net/EdemaGrading.html)

contact for an extended period of time and under different conditions, while the healthcare challenges are how to achieve a high sensitivity, accuracy, and specificity for detecting abnormal events in real-time.

Until recently, continuous monitoring of physiological parameters was possible only in the clinical settings. Nowadays, however, with developments in wearable sensors and personal technology, the possibility of continuous, real-time monitoring of physiological signals in the everyday context has become a reality. Especially, physiological monitoring has benefited significantly from advanced sensor capabilities, smart materials and miniature sensors, low-power computing and networking, and flexible circuits to integrate into the fabric and wearable items [18]. Researchers have investigated the development of wearable systems to unobtrusively and continuously monitor various physiological signals to improve personalized healthcare. Examples include a ring sensor capable of monitoring heart rate and oxygen in the blood [3], an ear-worn, flexible, lowpower sensor for long-term monitoring of heart rate [21], and a smart wristband for real-time perspiration analysis [13].

Contrary to the advancement in wearable technology and applications for health management in general, the domain of systematic edema assessment has received relatively little attention to date. Only recently, a few studies have attempted to develop wearable technologies to systematically assess edema using stretch sensors. These include Smart-Cuff, a wearable platform that continuously monitors edema real-time in remote and in-home settings [12], SmartSock, a wearable system that is equipped with accelerometer and flexible stretch sensor embedded in clothing for edema quantification [11], and the DEAP sensor, a wearable using dielectric electro-active polymer stretch sensors for continuous monitoring of edema [32]. The measurement approach of these systems is identical: they all attempted to assess edema 
by measuring a circumference of an ankle or foot using a stretch sensor.

\subsection{Challenges in Wearables for Healthcare}

Several technical challenges were acknowledged to overcome in the successful development of wearable systems for healthcare, especially with regard to data mining and signal processing to demonstrate the system's feasibility and analyze data [4] Amongst, we identified that two specific challenges are relevant to the scope of this paper: data preprocessing and data acquisition.

2.3.1. Data Processing. Although wearable sensors have shown promising results, additional work for data mining and processing of the raw sensor data is necessary to achieve desired and intended outcomes. In particular, a preprocessing of raw sensor data is essential due to the inevitable occurrence of noise, motion artifacts, and sensor errors in any wearable sensor systems. Preprocessing in wearable systems for healthcare involves filtering high frequency data and artifacts [4]. Commonly used techniques to remove high frequency noise from raw sensor data include power spectral density (PSD), fast Fourier transforms (FFT), and low-pass/high-pass filtering tools [23]. To filter artifacts, threshold-based methods to filter sensor data (e.g., [16,31]) or statistical tools to interpolate the missing data points (e.g., [1]) are commonly used.

2.3.2. Data Acquisition. In any health monitoring systems, having a robust data processing stage requires adequate amount of data to test the feasibility of a system. However, it is difficult to obtain real-world healthcare dataset due to ethical and technical difficulties. Therefore, many projects that developed health-monitoring systems used their own data gathering experiments to design, model, and test the data analysis step (e.g., [14,26]). In such cases, the gathered data are usually obtained by the predefined scenarios to evaluate the performed results.

Several studies used existing clinical datasets to test the data analysis step (e.g., [8,24]). Two commonly used online database are PhysioNet database [15] that consists a wide range of physiological data with categorized and robust annotations for complex clinical signals (e.g., $[1,17]$ ) and MIT data sets that contain the time series of patients' vital signs obtained from hospital medical information systems (e.g., [9,20]).

Lastly, some studies evaluated the performance of their systems through simulated data (e.g., [29]). Feasibility testing and data analysis using simulated data would be useful when the focus of data processing method is on the efficiency and robustness of information extraction rather than handling real-world data including the artifact, errors, or conditions of data gathering environment [30]. Another reason to use simulated data is when it is difficult to obtain real, large-scale data sets [33]. Because it is difficult to obtain a large-scale, real-world edema dataset, we used a simulated data to test the feasibility of Swellfit.

\section{System design and implementation}

We developed SwellFit, an experimental prototype of a wearable system that enables a patient-in an outpatient setting - to monitor peripheral edema with minimal effort and no expertise. SwellFit is a personal, unobtrusive, wearable system that monitors ankle swelling by measuring changes in the curvature of an ankle. SwellFit enables continuous, more accurate and reliable, less variable, and more sensitive measurement of ankle swelling with little effort and expertise compared to conventional measurements. The form of SwellFit is a traditional sweatband or a wrist brace in which an array of sensors and hardware are packaged (Figure 2). SwellFit consists of three parts: a hardware sensing platform (a wearable anklet), a mobile application for data visualization (an iPod Touch), and a web server with database for processing and storing data (Figure 3).

SwellFit continuously monitors the changes in ankle curvature for two reasons: an ankle diameter varies by person so that one can only detect edema by tracking relative changes in the value in a continuous manner, and continuous monitoring increases the chance to detect a signal of edema in time.

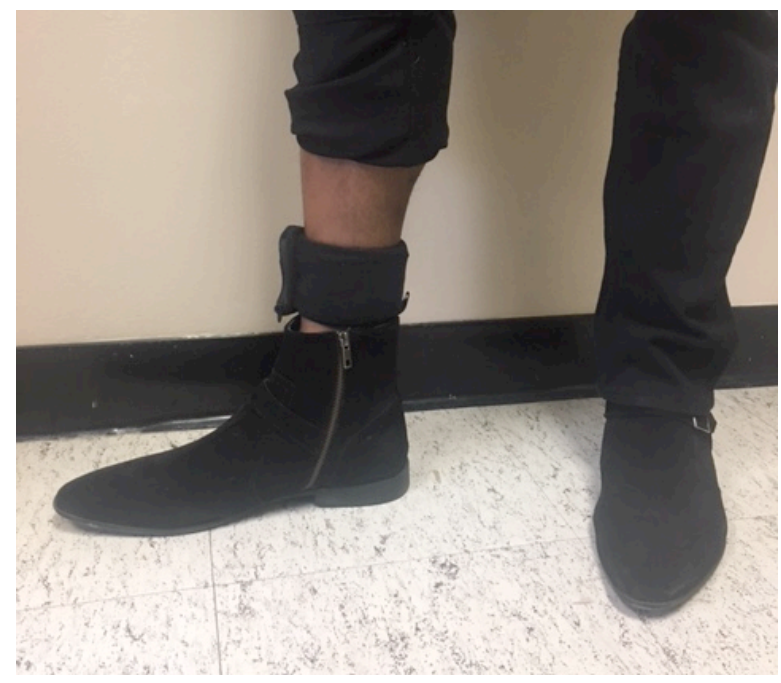

Figure 2. Final SwellFit implementation. The form factor of SwellFit is similar to a sweatband. 


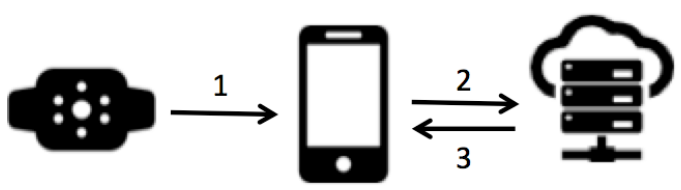

Figure 3. Data transfer between parts: SwellFit hardware, an iPod Touch and a webserver (from left to right). First, sensor readings from SwellFit are transferred to an iPod Touch via Bluetooth (1). Then, an iPod Touch sends the data to a server for signal processing through Wi-Fi (2). Finally, an iPod Touch receives the data for visualization from the server (3)

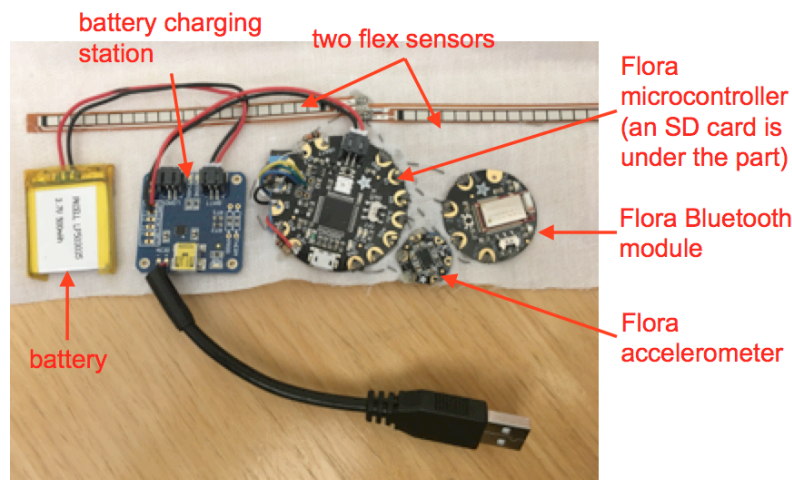

Figure 4. SwellFit hardware. Sensors and hardware parts are connected using conductive thread (except connections to a battery charging station) on a fabric substrate to implement a flexible wearable system that ensures comfort in wearing it and does not interfere with a range of human motion

\subsection{Hardware}

SwellFit hardware comprises six parts: two flex sensors, a Flora microcontroller with an SD memory card for onboard data storage, a Bluetooth 4.0 LE module, a Flora accelerometer, and a 500mAh battery with a battery charging circuit (Figure 4). A flex sensor detects bending: when a flex sensor is bent, its resistance changes from $10 \mathrm{k} \Omega$ to about $20 \mathrm{k} \Omega$. Two flex sensors are connected to wrap around an ankle, resulting in resistance changes from $20 \mathrm{k} \Omega$ to $40 \mathrm{k} \Omega$. Our system monitors this resistance change by measuring the voltage output of a voltage divider with a $10 \mathrm{k} \Omega$ reference. The output of the voltage divider is sampled at $200 \mathrm{~Hz}$ and the average value is recorded every second, resulting in an effective $1 \mathrm{~Hz}$ sampling rate. Sensor readings stored in the SD card are transmitted to a mobile application via Bluetooth when the mobile application is launched. Transmitted data include timestamp, raw flex-sensor values, and battery values. The battery values are sent to the mobile application so that it displays an indicator for remaining battery percentage.

\subsection{Mobile application}

A mobile application running on an iPod Touch is a platform for data transfer between the hardware (a wearable sensor) and a web server, as well as for data visualization. As soon as a mobile application receives new data from the hardware via Bluetooth, it transmits the data to a web server for signal processing via Wi-Fi (to be described in the next section). Then, swelling values processed in the backend are transmitted back to the mobile application to display the current swelling status and render changes in ankle swelling as a graph. In addition, the mobile application sends a push notification when the system detects an abnormal signal of swelling or when the battery in the wearable sensor is low. This paper primarily focuses on demonstrating the feasibility of SwellFit for distinguishing swelling from ankle curvature values using the flex sensor's readings. Thus, the design and implementation of a mobile application is not within the scope of this paper.

\subsection{Signal processing}

Once a new dataset comes from a mobile application into our database, a code is invoked to run three stages of signal processing in sequence, including: (1) cropping outliers, (2) removing noisy data, (3) finding high-and determining severity of swelling.

3.3.1. Cropping outliers. Because SwellFit uses an onboard voltage reference and 10-bit encoder, collected data can include outliers that do not make sense given the specification of a flex sensor, caused by voltage sag of the reference or a sensor misread from the encoder. Thus, the first step of signal processing is to identify and remove these outliers. According to the hardware specification, the resistance of two flex sensors connected in series ranges from $20 \mathrm{k} \Omega$ when flat to about $40 \mathrm{k} \Omega$ when flexed all the way. We used a $10 \mathrm{k} \Omega$ resistor in series with flex sensors to form a voltage divider with the onboard voltage reference of $3.3 \mathrm{~V}$ so that output voltage range results in from $2.2 \mathrm{~V}$ to $2.6 \mathrm{~V}$, as in:

$$
V_{\text {output }}=\frac{Z_{\text {sensor }}}{Z_{\text {sensor }}+Z_{\text {resistor }}} \cdot V_{\text {reference }}
$$

As our analog-to-digital converter represents $0 \mathrm{~V}-$ $3.3 \mathrm{~V}$ to $0-1023$ in 10 bits, $2.2 \mathrm{~V}$ maps to 680 bits and $2.6 \mathrm{~V}$ maps to 820 bits. Thus, we set the boundary of feasible sensor values between 680 and 820 , and considered any values outside of this boundary as 
outliers and replaced the value with the mean value of the whole dataset. This way we can ensure that the length of the dataset remained equal to the raw data without resampling. Through this first stage of signal processing, the system successfully removes outlier data from the dataset (Graph 1).

3.3.2. Filtering out noise data. Flex sensor readings can include artifacts caused by external forces other than ankle swelling. We identified three kinds of artifacts: motion artifacts (e.g., when a user unintentionally touches the sensor), rotational artifacts (e.g., when the sensor rotates or moves itself around an ankle), and human-interaction artifacts (e.g., when a user puts the sensor on and off an ankle). Thus, the next step of signal processing is to filter out these artifacts from our dataset.

The onset of edema is on the time scale of hours, whereas the aforementioned artifacts occur on the time scales of minutes or seconds. Thus, we applied a lowpass filter that keeps slow gradual changes but rejects rapid changes in the dataset. Specifically, we used a Butterworth filter, a digital signal-processing filter designed to have a flat frequency response in the passband with no ripple in the roll-off [25]. There are two values to adjust the roll-off of the Butterworth filter: a filter order and a cutoff frequency. Filter order determines how steep the roll-off should be. And, the cutoff frequency determines the frequency in which the roll-off begins. For a filter order, we found the 1st and 5th order filters to be too modest and too aggressive respectively. Because we are blind to incoming data, we decided to approach it moderately using the 3rd order filter. For a cutoff frequency $(\mathrm{Fc})$, we chose $0.015 \mathrm{~Hz}$ to discard values which fundamental frequency is less than a minute. Through this process, the system successfully removes the signal fluctuations occurring at the time scales of minutes or seconds, while keeping slower changes (Graph 2).

3.3.3. Distinguishing severity of swelling. As mentioned in the previous section, the common clinical approach to measuring edema is a subjective assessment of edema pitting, where an examiner visually checks severity of swelling using a score from 1 to 4 [7]. Following the scale of conventional edema pitting, we decided to assess severity of swelling using a score from 1 to 4 . To calculate a severity score, the system first calculates a baseline (a non-swelling state) by averaging the first 120 largest values (data collected for 4 minutes) in the trusted region of incoming data and the first 120 largest values in the trusted region of existing data. Throughout several iterations of experiments, we identified that data collected for minutes are large enough to effectively determine a

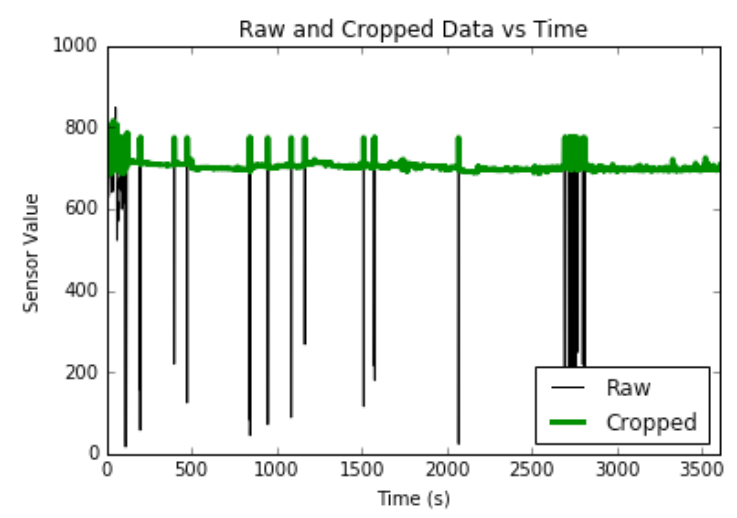

Graph 1. This section of raw data contains a lot of outliers (black vertical lines) caused by voltage sag. By cropping outliers that are out of the value boundary, the system removes the data that do not make sense.

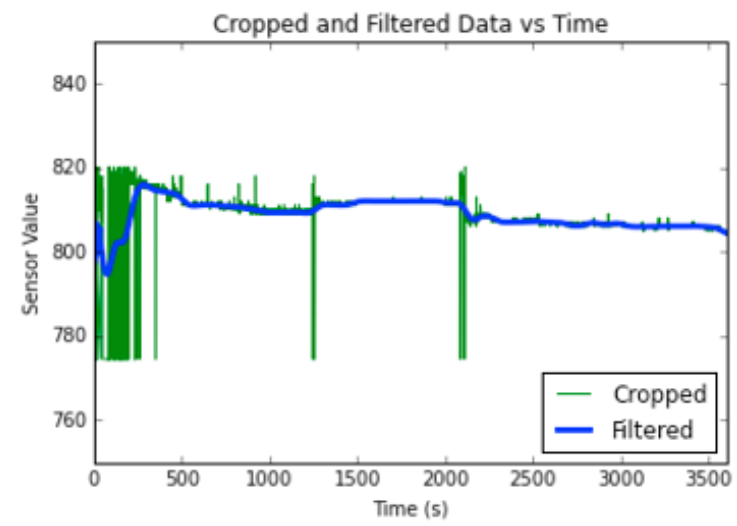

Graph 2. This section of cropped data (a green line) contains motion artifacts (sensor value change caused by artifacts) in the first 400 seconds, and at 1250 and 2100 seconds. Our signal-processing algorithm distinguishes these as noisy data and filters them out (a blue line).

baseline region. The system uses the largest values to calculate a baseline because a higher value in the flex sensor readings means less sensor bending and thus less swelling (e.g., If an ankle swells, the curvature of an ankle becomes flatter and a flex sensor bends less which cause value in the flex sensor readings to reduce). We use these 240 values (120 incoming/existing values each) to calculate a consistent baseline average.

In some occasions, the first 120 largest data readings may already contain values that are caused by substantial edema. In this case, the calculated baseline may not accurately reflect the actual non-edema state. Because we measure relative changes in ankle curvature in a continuous manner without an absolute ground truth of a non-edema sate, this potential bias in calculating a baseline is inevitable and we acknowledge this as a limitation of our system. 
However, once a user wears SwellFit for an extended period of time, such as for an hour or longer, this bias will decrease over time and reliability to capture a true non-edema state for a baseline will increase.

Using the calculated baseline as an estimate of a non-edema state, the system distinguishes edema as a scale of $5 \%$ decrease in the sensor value: up to $5 \%$ decrease maps to score 1 (no edema), 10\% maps to 2 (mild), 15\% maps to 3 (moderate), and 20\% maps to 4 (severe). For example, a 5\% decrease in the flex sensor reading approximately maps to a $2 \mathrm{~mm}$ increase in ankle diameter for someone with an ankle radius of $40 \mathrm{~mm}$. This scale complies with the degree to which a common pitting measurement uses [13]. We set $5 \%$ as a temporary but legitimate scale to detect swelling based on our dataset, because no clinical dataset exist to translate the volume of an ankle (circumference or curvature) into edema stages [18] Though, this scale needs to be adjusted, reflecting clinical expertise and further validations using a larger dataset.

\section{Feasibility testing}

We conducted two experiments to test the feasibility of the SwellFit prototype from two aspects: how it distinguishes noisy data (changes in sensor values caused by other factors than ankle swelling) from raw sensor values, and how it detects swelling with different severity levels. These experiments aim to provide a proof-of-concept demonstration of SwellFit in terms of the feasibility and accuracy of edema detection from continuous ankle curvature measurements.

\subsection{Collecting data from human subjects with artifacts}

We recruited four healthy adults (Table 1) to collect ankle curvature data using SwellFit. Each subject wore SwellFit for four consecutive days except when charging the battery. The subjects' activity levels varied: one subject engaged in a lot of activities including playing soccer, going for a bike ride and rock climbing, another subject walked a lot to commute, and the other two were pretty sedentary with little activity during the data collection period.

We did not formally assess the user experience of wearing SwellFit, but asked participants for brief feedback and personal reflection on wearing it at the end of data collection. Overall, the responses were positive in that wearing SwellFit was not intrusive or interfering with performing daily activities. All subjects but one mentioned that they almost forgot they were wearing it, whereas Subject 1 (with high activity level) commented that he felt warm around the ankle when he engaged in activities.

We processed the collected data using our signalprocessing algorithms to evaluate how SwellFit filters out noisy data from raw values to detect edema. Not surprisingly, we did not identify any signal of edema from the entire dataset, which makes sense because the subjects did not have any swelling condition. In the following, we illustrate the process through which raw sensor values are turned into edema scores in detail with two exemplar datasets.

\begin{tabular}{|c|c|c|c|}
\hline Subject & Gender & Age & Activity Level \\
\hline 1 & M & Mid 20s & Very active \\
\hline 2 & M & Early 30s & Walking a lot \\
\hline 3 & F & Late 30s & Sedentary \\
\hline 4 & M & Late 20s & Sedentary \\
\hline
\end{tabular}

Table 1. Subjects for the first set of data collection and their gender, age, and activity level

4.1.1. Case 1. The flex sensor values with high activity levels. The first dataset was collected while Subject 1 was playing soccer for an hour. In this dataset, he was in the mixed states of walking, running and standing (from 0 to 1500 seconds in the graph 3 ), and then running briskly (from 1500 to 2500 seconds) before returning to mild walking and standing (after 2500 seconds).

In the raw data (a black line in Graph 3, most of which is overlapped by the cropped data), there are two outliers caused by voltage sag. The first stage of signal processing successfully cropped these outliers from the data. Next, the data are smoothed by filtering out abrupt changes in the raw data that occurred on the order of minutes, which we identify as artifacts using the Butterworth filter (Graph 4).

Finally, we distinguish if edema occurs by calculating the rate of decrease in the trusted data from the baseline. When the decrease rate is over $5 \%$, we consider it as edema. This region of data is plotted in green, meaning no sign of notable swelling (Graph 5). In all, our signal-processing algorithm successfully cropped outliers and filtered heavy motion artifacts without throwing out useful information.

4.1.2. Case 2. The flex sensor values with low activity levels. The second dataset was collected while Subject 2 was walking. In this data, he went from a walking to a sedentary state. In the raw data, there were a few outliers in the beginning of data collection. The first stage of signal processing successfully cropped these outliers from the data (Graph 6). Next, the data are again smoothed through filtering out 
abrupt changes in the raw values that occurred on the order of minutes and seconds, which we identify as artifacts using the Butterworth filter.

Contrary to the filtered data in Graph 4, this filtered data did not have any spine except a small inversed spine in the plot, meaning that this dataset does not have many motion artifacts (Graph 7).

Finally, we distinguish if edema happens by calculating the rate of decrease in the trusted data from the baseline. In this data, ankle curvature gradually

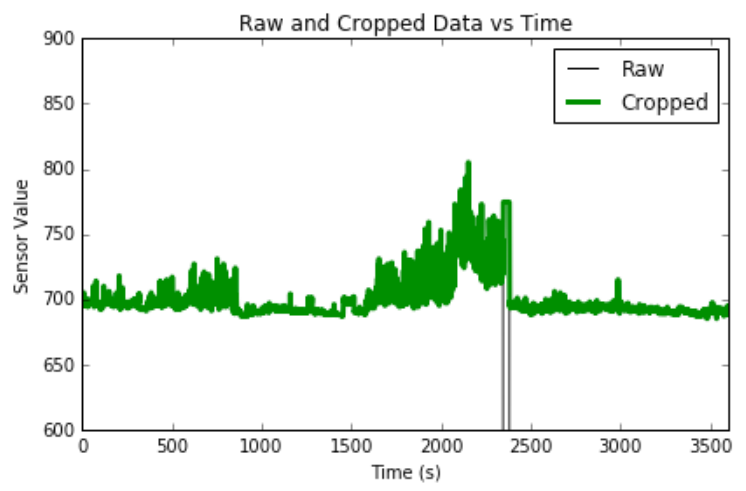

Graph 3. Cropped data from the raw flex sensor values

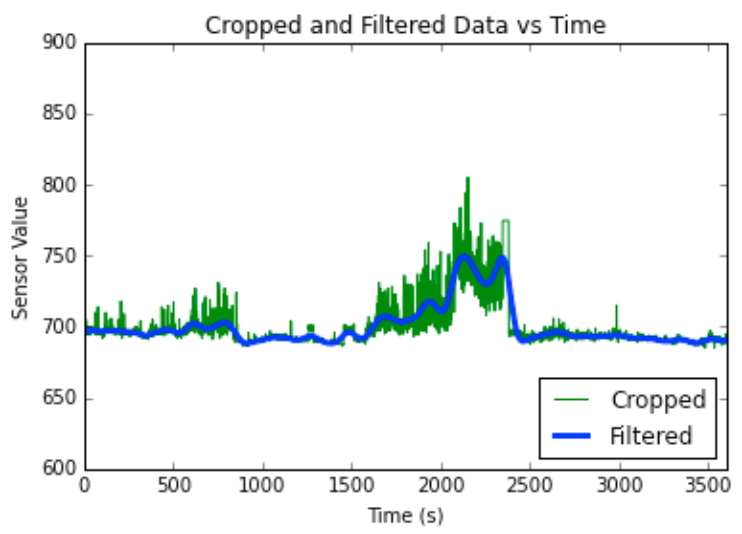

Graph 4. Filtered data from the cropped data

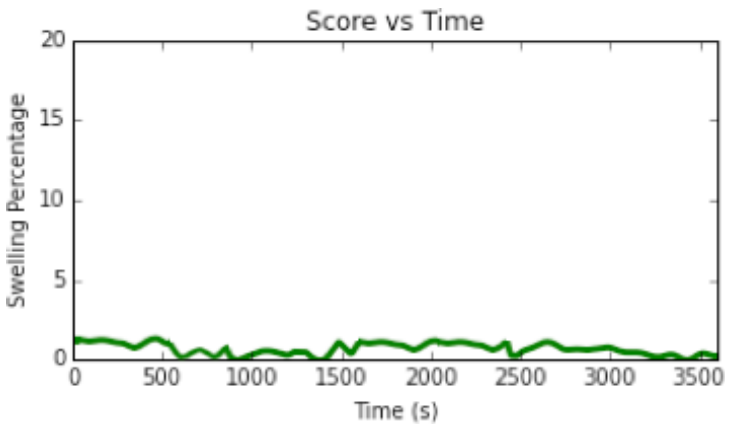

Graph 5. Calculated swelling percentage from the trusted data. There is no sign of swelling in this region of the data decreases, which makes sense because sitting for an extended period of time causes feet to swell mildly (recall that a higher flex sensor value means less sensor bending and less swelling). However, the decrease rate in the trusted data was less than $1 \%$ so that we do not consider it as actual edema unless more data come in with further signs of edema (Graph 8). In all, again, the signal-processing algorithm successfully cropped outliers and filtered heavy motion artifacts without throwing out useful information.

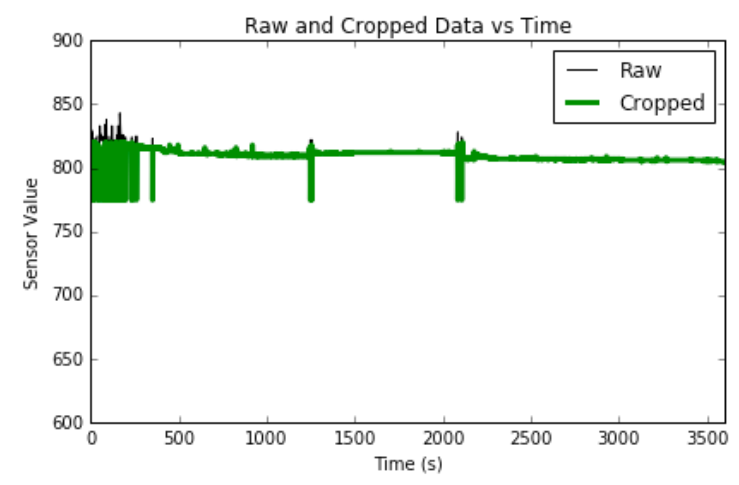

Graph 6. This section of data shows cropped data from the original raw flex sensor values

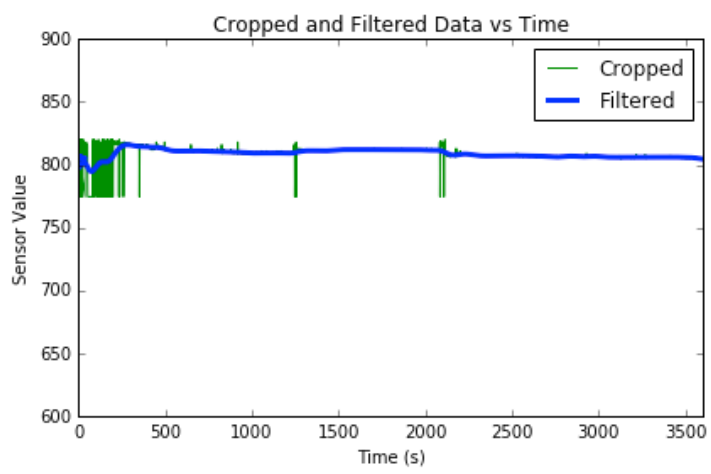

Graph 7. This section of data shows filtered data from the cropped data

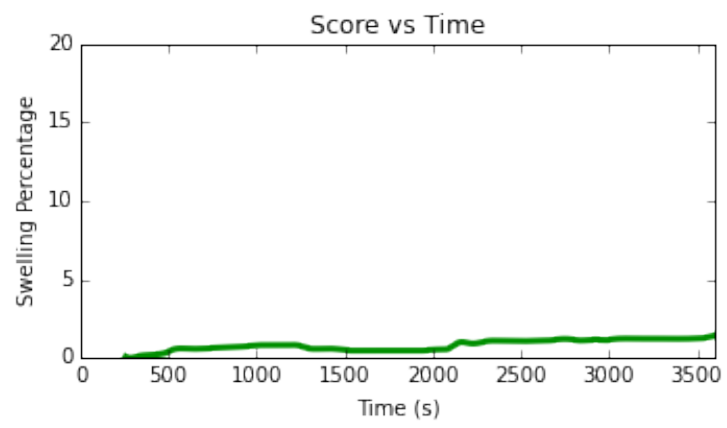

Graph 8. Calculated swelling percentage from the trusted data. There is no sign of swelling in this region of the data. 


\subsection{Simulating Swelling}

As we currently do not have access to patients with edema conditions, we simulated swelling using a balloon by increasing its air pressure gradually to test how SwellFit performs. We changed the air pressure of a balloon as gradually as possible in a steady condition to simulate swelling. Thus, no outliers and noisy data were introduced in the dataset, which resulted in the trusted data being almost identical to the raw flex sensor values (Graph 9). As expected, the sensor value gradually decreases as the balloon is inflated.

We calculated the rate of decrease in the trusted data from the baseline to distinguish edema with different levels of severity. Our system successfully distinguished edema with different severity levels: when the decrease in the trusted data exceeds $5 \%$ from the baseline, the color of the plot line changes to yellow to indicate mild edema. When the rate exceeds $10 \%$, the color of the plot line changes to orange to indicate moderate edema. When the rate exceeds $15 \%$, the color of the plot line changes to red to indicate severe edema (Graph 10). We iterated collecting the simulated swelling data using a balloon four times, and

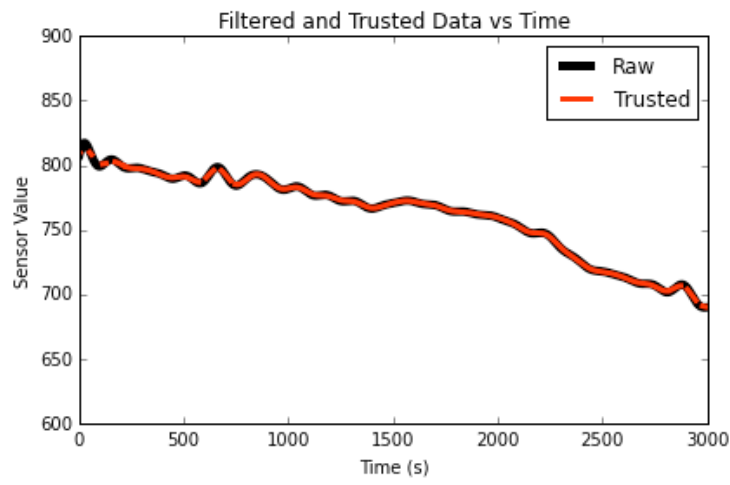

Graph 9. This section of data shows that the trusted data after signal processing is identical to the raw values.

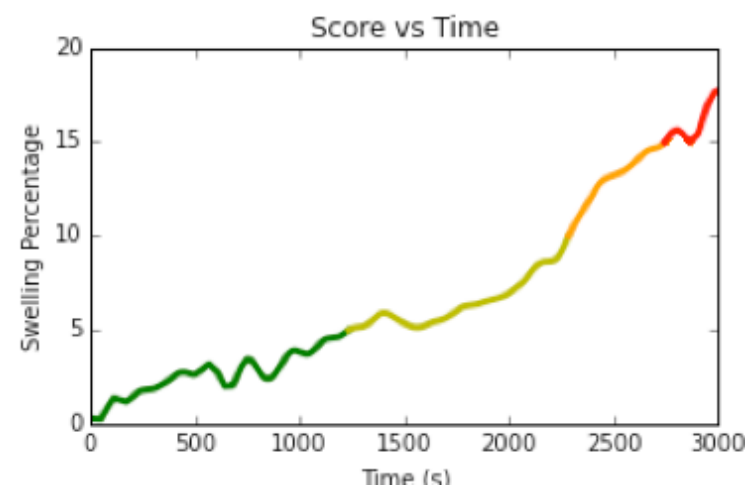

Graph 10. Calculated swelling percentage from the trusted data. The color of the plotted line changes from green to yellow to orange to red depending on the severity of swelling. the results were identical across all four datasets.

\subsection{In Summary}

The data from our evaluation with four healthy participants demonstrated the proof of concept of SwellFit: it did not detect edema where none was present even in the presence of vigorous real-world physical activity, which was a source of substantial noise in the raw sensor measurements. The data from the evaluation with the balloon demonstrated the feasibility of the system: as the balloon's diameter increased slowly over a period of 3000 seconds, SwellFit correctly detected abnormal swelling.

\section{Limitations and future work}

Through a series of the proof-of-concept studies, this paper successfully demonstrates the feasibility of the experimental SwellFit system that can assess edema through continuous monitoring of the changes in ankle curvature. However, we acknowledge that our system implementation and its feasibility testing have limitations. First, SwellFit monitors changes in ankle curvature in a continuous manner without any absolute ground truth data of non-edema. Because of this, the calculated baseline may not accurately reflect the actual non-edema state if the data already contain substantial edema or artifacts. Thus, SwellFit works best when a user wears it for an extended period of time to capture the actual non-swelling status.

Another limitation is that we did not conduct a performance evaluation with people who have edema conditions. However, simulated swelling using a balloon is mechanically identical to actual swelling, and thus we believe our experiments still convey meaningful outcomes as a proof-of-concept simulation. The question is that we have not tested our system with data that contain both noisy data and swelling. In theory, our system will still work well because we created layers of the signal processing filters that are robust enough to distinguish swelling from such messy data. However, statistical analysis of the performance testing with a sufficient number of human subjects which data contain both swelling and noisy data is a required next step to ensure its performance.

\section{References}

[1] Apiletti, Daniele, Elena Baralis, Giulia Bruno, and Tania Cerquitelli. "Real-time analysis of physiological data to support medical applications." IEEE transactions on information technology in biomedicine (2009): 313-321.

[2] Armer, Jane M., M. Elise Radina, Davina Porock, and Scott D. Culbertson. "Predicting breast cancer-related lymphedema 
using self-reported symptoms." Nursing research 52, no. 6 (2003): 370-379.

[3] Asada, H. Harry, Phillip Shaltis, Andrew Reisner, Sokwoo Rhee, and Reginald C. Hutchinson. "Mobile monitoring with wearable photoplethysmographic biosensors." IEEE engineering in medicine and biology magazine 22 , no. 3 (2003): 28-40.

[4] Banaee, Hadi, Mobyen Uddin Ahmed, and Amy Loutfi. "Data mining for wearable sensors in health monitoring systems: a review of recent trends and challenges." Sensors 13, no. 12 (2013): 17472-17500.

[5] Bonato, Paolo. "Advances in wearable technology and applications in physical medicine and rehabilitation." Journal of neuroengineering and rehabilitation 2, no. 1 (2005): 2.

[6] Bonato1, Paolo. "Wearable sensors and systems." IEEE Engineering in Medicine and Biology Magazine 29, no. 3 (2010): 25-36.

[7] Brodovicz, Kimberly G., Kristin McNaughton, Naoto Uemura, Gary Meininger, Cynthia J. Girman, and Steven H. Yale. "Reliability and feasibility of methods to quantitatively assess peripheral edema." Clinical medicine \& research 7, no. 1-2 (2009): 21-31.

[8] Clifton, Lei, David A. Clifton, Marco AF Pimentel, Peter J. Watkinson, and Lionel Tarassenko. "Gaussian processes for personalized e-health monitoring with wearable sensors." IEEE Transactions on Biomedical Engineering 60, no. 1 (2013): 193197.

[9] Desai, Akshay S., and Lynne W. Stevenson. "Rehospitalization for heart failure predict or prevent?." Circulation 126, no. 4 (2012): 501-506.

[10] Ding, H.; Sun, H.; mean Hou, K. Abnormal ECG Signal Detection Based on Compressed Sampling inWearable ECG Sensor. In Proceedings of the International Conference onWireless Communications and Signal Processing, Nanjing, China, 9-11 November 2011; pp. 1-5.

[11] Fallahzadeh, Ramin, Mahdi Pedram, and Hassan Ghasemzadeh. "Smartsock: A wearable platform for contextaware assessment of ankle edema." In Engineering in Medicine and Biology Society, 2016 IEEE 38th Annual International Conference of the, pp. 6302-6306. IEEE, 2016.

[12] Fallahzadeh, Ramin, Mahdi Pedram, Ramyar Saeedi, Bahman Sadeghi, Michael Ong, and Hassan Ghasemzadeh. "Smart-cuff: A wearable bio-sensing platform with activity-sensitive information quality assessment for monitoring ankle edema." In Pervasive Computing and Communication Workshops, pp. 57-62. IEEE, 2015.

[13] Gao, Wei, Sam Emaminejad, Hnin Yin Yin Nyein, Samyuktha Challa, Kevin Chen, Austin Peck, Hossain M. Fahad et al "Fully integrated wearable sensor arrays for multiplexed in situ perspiration analysis." Nature 529, no. 7587 (2016): 509-514.

[14] Gaura, Elena, John Kemp, and James Brusey. "Leveraging knowledge from physiological data: On-body heat stress risk prediction with sensor networks." IEEE transactions on biomedical circuits and systems 7, no. 6 (2013): 861-870.

[15] Goldberger, Ary L., Luis AN Amaral, Leon Glass, Jeffrey M. Hausdorff, Plamen Ch Ivanov, Roger G. Mark, Joseph E. Mietus, George B. Moody, Chung-Kang Peng, and H. Eugene Stanley. "Physiobank, physiotoolkit, and physionet." Circulation 101, no. 23 (2000): e215-e220.

[16] Lee, Han-Wook, Ju-Won Lee, Won-Geun Jung, and Gun-Ki Lee. "The periodic moving average filter for removing motion artifacts from PPG signals." International Journal of Control, Automation, and Systems 5, no. 6 (2007): 701-706.

[17] Lloyd-Jones, Donald, et al. "Heart disease and stroke statistics-2009 update a report from the American Heart Association Statistics Committee and Stroke Statistics Subcommittee." Circulation 119.3 (2009): e21-e181

[18] Mao, Yi, Wenlin Chen, Yixin Chen, Chenyang Lu, Marin Kollef, and Thomas Bailey. "An integrated data mining approach to real-time clinical monitoring and deterioration warning." In Proceedings of the 18th international conference on Knowledge discovery and data mining, pp. 1140-1148. ACM, 2012.

[19] O’Sullivan, S. B. "Stroke In: O’Sullivan SB and Schmitz TJ." Physical Rehabilitation. FA Davis, Philadelphia, PA (2007): 706-776.

[20] Patel, Abhilasha M., Pankaj K. Gakare, and A. N. Cheeran. "Real time ECG feature extraction and arrhythmia detection on a mobile platform." Int. J. Comput. Appl 44, no. 23 (2012): 4045.

[21] Patel, Shyamal, Hyung Park, Paolo Bonato, Leighton Chan, and Mary Rodgers. "A review of wearable sensors and systems with application in rehabilitation." Journal of neuroengineering and rehabilitation 9, no. 1 (2012): 21.

[22] Patterson, James AC, Douglas C. McIlwraith, and GuangZhong Yang. "A flexible, low noise reflective PPG sensor platform for ear-worn heart rate monitoring." In Wearable and Implantable Body Sensor Networks, 2009. BSN 2009. Sixth International Workshop on, pp. 286-291. IEEE, 2009.

[23] Raju, Seshadri, Sam Owen, and Peter Neglen. "The clinical impact of iliac venous stents in the management of chronic venous insufficiency." Journal of vascular surgery 35 , no. 1 (2002): 8-15.

[24] Reddy, K. Ashoka, Boby George, and V. Jagadeesh Kumar. "Use of fourier series analysis for motion artifact reduction and data compression of photoplethysmographic signals." IEEE Transactions on Instrumentation and Measurement 58 , no. 5 (2009): 1706-1711.

[25] Salem, Osman, Yaning Liu, and Ahmed Mehaoua. "A lightweight anomaly detection framework for medical wireless sensor networks." In Wireless Communications and Networking Conference, 2013 IEEE, pp. 4358-4363.

[26] Selesnick, I. W., \& Burrus, C. S. (1998). Generalized digital Butterworth filter design. Signal Processing, IEEE Transactions on, 46(6), 1688-1694

[27] Silva, Fábio, Teresa Olivares, Fernando Royo, M. A. Vergara, and Cesar Analide. "Experimental study of the stress level at the workplace using an smart testbed of wireless sensor networks and ambient intelligence techniques." In International Work-Conference on the Interplay Between Natural and Artificial Computation, pp. 200-209. Springer, Berlin, Heidelberg, 2013.

[28] Singh, Rajiv Ranjan, Sailesh Conjeti, and Rahul Banerjee. "An approach for real-time stress-trend detection using physiological signals in wearable computing systems for automotive drivers." In Intelligent Transportation Systems, 2011 14th International IEEE Conference on, pp. 1477-1482.

[29] Vu, Thi Hong Nhan, Namkyu Park, Yang Koo Lee, Yongmi Lee, Jong Yun Lee, and Keun Ho Ryu. "Online discovery of Heart Rate Variability patterns in mobile healthcare services." Journal of Systems and Software 83, no. 10 (2010): 1930-1940.

[30] Wang, Wei, Honggang Wang, Michael Hempel, Dongming Peng, Hamid Sharif, and Hsiao-Hwa Chen. "Secure Stochastic ECG Signals Based on Gaussian Mixture Model for eHealthcare Systems." IEEE Systems Journal 5, no. 4 (2011): 564-573.

[31] Wood, Levi Benjamin. "Motion artifact reduction for wearable photoplethysmogram sensors using micro accelerometers and Laguerre series adaptive filters." PhD diss., Massachusetts Institute of Technology, 2008.

[32] Yu, Fei, Arne Bilberg, Lin Xiao, and Knud Bonnet Yderstraede. "Foot edema simulation and monitoring using dielectric electro-active polymer sensors." Sensors and Actuators A: Physical (2015): 33-40.

[33] Zhu, Ying. "Automatic detection of anomalies in blood glucose using a machine learning approach." Journal of Communications and Networks 13, no. 2 (2011): 125-1 